\title{
Ready for avian flu?
}

\section{Committing to a vaccine stockpile is just the beginning. Tadataka Yamada, Alice Dautry and Mark Walport offer a roadmap for heading off a global avian influenza catastrophe.}

$\bigcirc$ everal chilling considerations highlight the seriousness of an impending pandemic of the H5N1 'avian' influenza virus. The highly contagious nature of influenza, the limited ability to restrict its transmission and the efficiency of modern international transport all conspire to reduce the time from the first infection to a potential global crisis. Recent models built on data from the 1918 flu pandemic predict that 50 million- 80 million people could $\mathrm{die}^{1}$. Perhaps not surprisingly, 95\% of these deaths are likely to occur in the developing world, where higher population density, poor health status and limited access to public-health interventions prevail. Prevention through vaccination would be optimal, but vaccines against a pandemic strain might take six months to manufacture and deliver, even in developed countries. Moreover, total global capacity for flu vaccine manufacture in the first 12 months is estimated at only 500 million doses, and no global financing-vehicle exists.

There is some good news. The World Health Organization (WHO) has announced plans to stockpile $\mathrm{H} 5$ influenza vaccine and several manufacturers have already offered to contribute. Other manufacturers are supportive but await details before committing. Efforts have been initiated by the WHO to create a policy framework for vaccine allocation and recommendations for its use. An ethics framework, and financing, regulatory and distribution systems will also have to be developed with member states.

Several recent developments make this stockpile feasible. H5N1 vaccines with adjuvants that reduce the required dose as much as fourfold have been developed ${ }^{2}$ and one has been licensed for medical use. Furthermore, the manufacturing capacity of 500 million doses is calculated on a requirement for three strains of flu virus for standard vaccinations; in crisis mode, three times as much monovalent pandemic flu vaccine could be produced. Together, these considerations could increase global vaccine production capacity to 5 billion-6 billion doses over 12 months. Moreover, adjuvant-enhanced vaccines may provide cross-protection against strains that have undergone up to seven years of genetic drift ${ }^{3}$. If this is true, appropriate planning, manufacture and stockpiling of currently effective vaccines might provide the basis for an immediate response to an H5N1 outbreak.

But this is not the time to be complacent.
Our organizations, the Bill \& Melinda Gates Foundation, the Pasteur Institute and the Wellcome Trust, are now planning, with major medical-research funders and other stakeholders, several projects to enhance the research effort and reduce the risks from the threat of pandemic influenza over coming decades. In the next 18 months we will develop, maintain and disseminate a central inventory of funded research activities that are relevant to human

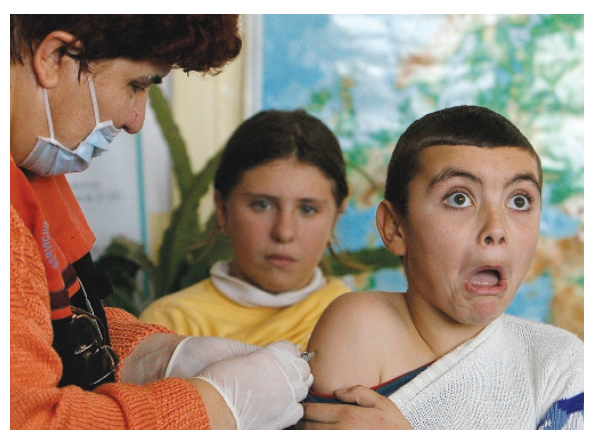

Vaccines: still the best defence against avian flu.

influenza to ensure that stakeholders are wellinformed. We will also coordinate roadmapping exercises to identify knowledge gaps. These will assist funders and researchers in establishing research-funding priorities, with specific focus on vaccines, drug therapies and epidemiology/ population science (for example, diagnostics, surveillance, transmission and modelling). The Bill \& Melinda Gates Foundation and the Wellcome Trust will collaborate to fund these activities.

These two programmes will enable the development of a cohesive health-research agenda for pandemic influenza. In addition, we call on the wider community to focus on the following challenges.

\section{Resolve intellectual-property claims}

We, as a community, must be transparent and open in sharing information, reagents, viral strains and scientific know-how to conduct and advance the best science and rapidly create the most effective pandemic vaccines. We must ensure that intellectual property concerns in either the developing or the developed world do not stand in the way of effective solutions.

\section{Coordinate stockpiling efforts}

We must seize the opportunity afforded by emerging science to mobilize the global capacity to manufacture an effective 'pre-pandemic'
H5-vaccine stockpile with novel vaccines currently under evaluation. Although the capacity to manufacture pandemic flu vaccines is large, we must continue to supply standard seasonal flu vaccine to those at risk.

\section{Consider all costs}

We must make sure that all people from all nations will benefit from stockpiling, while ensuring fair financing. This implies a tieredpricing model across countries. Careful consideration must also be given to providing adequate protection for health providers and manufacturers against costs of claims for unpredictable side-effects of vaccination.

\section{Initiate a funding mechanism}

Even at the lowest prices it will be difficult for many nations to meet the needs of their people. Public and private donors must create a financing facility to help the poorest nations pay for their share of the pandemic flu vaccine. Donors have funded financing mechanisms, such as the Geneva-based GAVI Alliance, which supports the purchase of childhood vaccines, to address other urgent health challenges.

\section{Bolster surveillance}

We must build robust mechanisms; for surveillance of an outbreak of pandemic flu and for delivering prevention and treatment, particularly vaccines, as quickly and broadly as possible. There must be full integration of vaccine strategies with other approaches, and we must coordinate research strategies for dealing with zoonotic and human influenza infections.

Advances in science and society can help prevent disaster if we resolve to act collectively. Tadataka Yamada is the executive director of the Bill \& Melinda Gates Foundation's Global Health Program, 1551 Eastlake Avenue East, Seattle, Washington 98102, USA. Alice Dautry is director general, Pasteur Institute, 25 -28 rue du Docteur Roux, 75724 Paris, France. Mark Walport is director of the Wellcome Trust, Gibbs Building 215 Euston Road, London NW1 2BE, UK.

1. Murray, C. J., Lopez, A. D., Chin, B., Feehan, D. \& Hill, K. H. Lancet 368, 2211-2218 (2006)

2. Leroux-Roels, I. et al. Lancet 370, 580-589 (2007).

3. Stephenson, I. et al. J. Infect. Dis. 191, 1210-1215 (2005).

Competing financial interests: declared (see online article for details)

See Editorial, page 137. To discuss this article online visit http://tinyurl.com/625swp. 